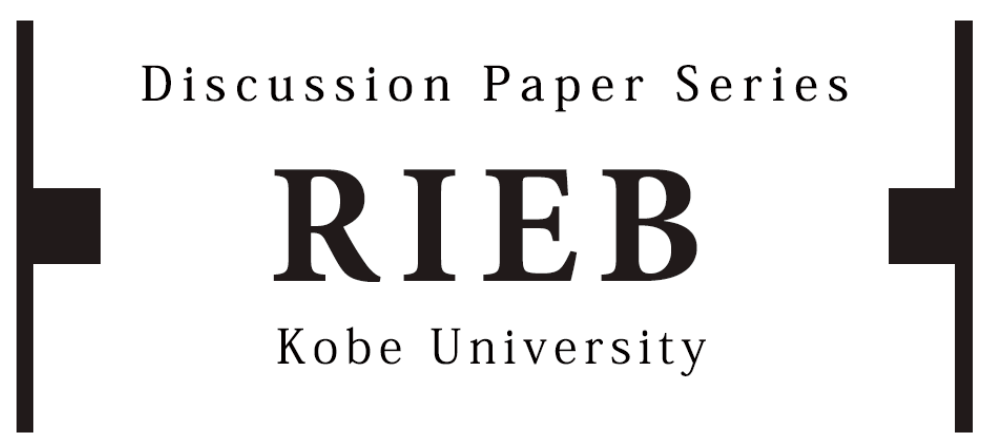

DP2011-20

\title{
Monotonicity and Continuity of the Critical Capital Stock in the Dechert-Nishimura Model
}

Ken-Ichi AKAO

Takashi KAMIHIGASHI

Kazuo NISHIMURA

Revised September 29, 2011

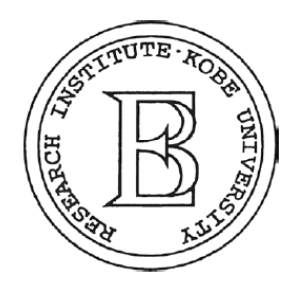

Research Institute for Economics and Business Administration

Kobe University 


\title{
Monotonicity and Continuity of the Critical Capital Stock in the Dechert-Nishimura Model $^{*}$
}

\author{
Ken-Ichi Akao ${ }^{\dagger}$ Takashi Kamihigashi ${ }^{\ddagger}$ Kazuo Nishimura ${ }^{\S}$ \\ September 29, 2011
}

\begin{abstract}
We show that the critical capital stock of the Dechert-Nishimura (1983) model is a decreasing and continuous function of the discount factor. We also show that the critical capital stock merges with a nonzero steady state as the discount factor decreases to a certain boundary value, and that the critical capital stock converges to the minimum sustainable capital stock as the discount factor increases to another boundary value.
\end{abstract}

JEL Classification: C61; D90; O41

Keywords: Dechert-Nishimura model; nonconvexity; optimal growth; critical capital stock; threshold

${ }^{*}$ We would like to thank an associate editor of this journal and an anonymous referee for their constructive criticisms and helpful suggestions. Financial support from the Japan Society for the Promotion of Science and the Joint Usage and Research Center, the Institute of Economic Research, Kyoto University, is gratefully acknowledged.

†School of Social Sciences, Waseda University, Japan. akao@waseda.jp

${ }^{\ddagger}$ Corresponding Author: RIEB, Kobe University, Japan. Tel/Fax: +81-78-803-7015. tkamihig@rieb.kobe-u.ac.jp

§KIER, Kyoto University, Japan. nishimura@kier.kyoto-u.ac.jp 


\section{Introduction}

Dechert and Nishimura (1983) studied a one-sector optimal growth model with a convex-concave production function, showing that any optimal (capital) path is monotone and converges to a steady state. One of their major findings is that there exists a unique critical capital stock such that any optimal path from an initial capital stock below this critical stock converges to zero, and any optimal path from an initial capital stock above this critical stock converges to a nonzero steady state. This threshold property is often associated with important economic phenomena such as history dependence and poverty traps.

Dechert and Nishimura's (1983) analysis has been extended by various studies on optimal growth with nonconvexities (e.g., Majumdar and Mitra, 1982; Majumdar and Nermuth, 1982; Mitra and Ray, 1984; Kamihigashi and Roy, 2007; Hung et al., 2009). The threshold property described above is also widespread even outside the optimal growth literature, arising in a broad range of economic problems concerning, for example, optimal investment for firms (e.g, Hartl and Kort, 2004; Haunschmied, et al., 2005; Wagener, 2005), renewable resources (e.g., Wirl, 2004), political behavior (e.g, Caulkins et al., 2001; Caulkins et al., 2007), and drug control (Tragler et al., 2001; Levy et al., 2006). A critical capital stock in such models is often referred to as a "Dechert-Nishimura-Skiba" point, after Dechert and Nishimura (1983) and Skiba (1978).

In terms of economic significance, the critical capital stock of the DechertNishimura model seems to be comparable to the nonzero steady state of the one-sector neoclassical growth model. The latter can be regarded as characterizing the long run behavior of an economy under neoclassical assumptions, and its comparative statics is one of the most powerful tools for economists in explaining cross-country differences. For example, it is well understood that a more patient country invests more and produces more in the long run, and countries with similar levels of patience have similar steady states. As for the critical capital stock of the Dechert-Nishimura model, although it is literally critical in determining whether the economy grows or shrinks, even its basic comparative statics properties are unknown in the current literature. For example, the literature does not offer the answers to the following basic questions: Does a more patient country have a smaller critical capital stock? Do countries with similar levels of patience have similar critical stocks?

The second question concerns parametric continuity. Without this prop- 
erty, a small change in a parameter could cause a sudden regime shift: after a small change, an economy in a poverty trap could suddenly start to grow, or a growing economy could suddenly start to shrink. Are such dramatic regime shifts possible?

In this paper we show that the critical capital stock of the DechertNishimura model is a decreasing and continuous function of one of the fundamental parameters, the discount factor. We use a comparative statics result shown by Amir et al. (1991) to establish that the critical capital stock is decreasing in the discount factor. This result means that a more patient country has a lower critical capital stock, and thus is more likely to escape from a poverty trap. A more significant contribution of this paper is to establish the continuity property of the critical capital stock. We do so by utilizing the value functions corresponding to the maximization problem with the additional constraint that feasible paths must be decreasing, and the one with the additional constraint that feasible paths must be increasing. This continuity result makes it clear that a small change in the discount factor does not cause a sudden regime shift and does not have a large impact on long run economic performance unless the economy is exactly at the critical capital stock.

In addition to the above results, we show that the critical capital stock converges to the "minimum sustainable capital stock" as the discount factor increases to a certain boundary value, and merges with a nonzero steady state as the discount factor decreases to another boundary value. If the discount factor decreases further, then the steady state disappears, and all optimal paths converge to zero.

The rest of the paper is organized as follows. In Section 2 we present the Dechert-Nishimura model under slightly weaker assumptions than in the original model. In Section 3 we collect various preliminary results to facilitate the proofs of our main results. In Section 4 we state our main results, which we prove in Section 5 . 


\section{The Model}

Consider the following maximization problem:

$$
\begin{aligned}
\max _{\left\{c_{t}, x_{t+1}\right\}_{t=0}^{\infty}} & \sum_{t=0}^{\infty} \beta^{t} u\left(c_{t}\right) \\
\text { s.t. } & c_{t}+x_{t+1}=f\left(x_{t}\right), \\
& c_{t}, x_{t+1} \geq 0 \\
& x_{0} \geq 0 \text { given, }
\end{aligned}
$$

where $c_{t}$ is consumption in period $t, x_{t}$ is the capital stock at the beginning of period $t, \beta \in(0,1)$ is the discount factor, $u$ is the utility function, $f$ is the production function, and (2.2) and (2.3) are required to hold for all $t \in \mathbb{Z}_{+}$. We maintain the following assumptions throughout the paper.

Assumption 2.1. $u: \mathbb{R}_{+} \rightarrow \mathbb{R}_{+}$is continuously differentiable on $(0, \infty)$, continuous, strictly increasing, strictly concave, and satisfies $u(0)=0$ and $\lim _{c \downarrow 0} u^{\prime}(c)=\infty$.

Assumption 2.2. $f: \mathbb{R}_{+} \rightarrow \mathbb{R}_{+}$is continuously differentiable on $(0, \infty)$, continuous, strictly increasing, and satisfies $f(0)=0$.

Assumption 2.3. There exists $x^{I}>0$ such that $f$ is strictly convex on $\left[0, x^{I}\right]$ and strictly concave on $\left[x^{I}, \infty\right)$.

Assumption 2.4. There exists $\bar{x}>x^{I}$ such that $f(\bar{x})=\bar{x}$ and $f(x)<x$ for all $x>\bar{x}$.

Unlike Dechert and Nishimura (1983), we do not assume that $u$ and $f$ are twice continuously differentiable. ${ }^{1}$ Assumption 2.3 means that $f$ is convex-concave, or S-shaped. Assumption 2.4 implies that all capital and consumption paths satisfying (2.2) and (2.3) are bounded. We call $\bar{x}$ the maximum sustainable capital stock. We also define the minimum sustainable capital stock $\underline{x}$ as follows:

$$
\underline{x}=\sup \{x>0: \forall y \in(0, x), f(y)<y\} .
$$

\footnotetext{
${ }^{1}$ However, Dechert and Nishimura's (1983) main arguments do not require $u$ and $f$ to be twice continuously differentiable.
} 
If there is no $x>0$ such that $f(y)<y$ for all $y \in(0, x)$, we define $\underline{x}=0$. If $\underline{x}>0$, then

$$
\forall x \in(0, \underline{x}), \quad f(x)<x .
$$

Thus any capital stock below $\underline{x}$ is not sustainable. Assumptions 2.2 and 2.4 together with (2.5) and (2.6) imply that

$$
\underline{x} \leq \bar{x}, \quad f(\underline{x})=\underline{x} .
$$

We use the following standard definitions. A feasible path (from $x \geq$ $0)$ is a capital path $\left\{x_{t}\right\}_{t=0}^{\infty}$ such that for some consumption path $\left\{c_{t}\right\}_{t=0}^{\infty}$, $\left\{c_{t}, x_{t+1}\right\}_{t=0}^{\infty}$ satisfies (2.2)-(2.4) for all $t \in \mathbb{Z}_{+}$(with $x_{0}=x$ ). An optimal path (from $x \geq 0$ ) is a feasible path $\left\{x_{t}\right\}$ (from $x$ ) such that for some consumption path $\left\{c_{t}\right\},\left\{c_{t}, x_{t+1}\right\}$ solves the maximization problem (2.1)-(2.4). A stationary path is a constant path. A capital stock $x \geq 0$ is a steady state if the stationary path $\left\{x_{t}\right\}$ such that $x_{t}=x$ for all $t \in \mathbb{Z}_{+}$is optimal.

\section{Preliminary Results}

In this section we present various preliminary results to facilitate the proofs of our main results.

\subsection{Basic Properties of Optimal Paths}

In this subsection we show some preliminary results after stating without proof several results from Dechert and Nishimura (1983) and Kamihigashi and Roy (2007) as well as their immediate consequences. ${ }^{2}$ One of the key results in this subsection is a proposition that summarizes Dechert and Nishimura's (1983) main conclusions on the critical capital stock.

By a standard argument, an optimal path from any $x \geq 0$ exists (see, e.g, Le Van and Morhaim, 2002, Theorem 1). For $x \geq 0$, let $v(x)$ be the

\footnotetext{
${ }^{2}$ There are similar and related results in the literature (e.g., Majumdar and Mitra, 1982; Majumdar and Nermuth, 1982; Mitra and Ray, 1984); see Kamihigashi and Roy (2007) for discussion of the literature.
} 
maximized value of the objective function:

$$
\begin{aligned}
v(x)=\max _{\left\{c_{t}, x_{t+1}\right\}_{t=0}^{\infty}} & \sum_{t=0}^{\infty} \beta^{t} u\left(c_{t}\right) \\
\text { s.t. } & c_{t}+x_{t+1}=f\left(x_{t}\right), \\
& c_{t}, x_{t+1} \geq 0, \\
& x_{0}=x .
\end{aligned}
$$

The value function $v$ satisfies the Bellman equation

$$
v(x)=\max _{y \in[0, f(x)]}\{u(f(x)-y)+\beta v(y)\} .
$$

Let $K$ denote the (optimal) policy correspondence:

$$
K(x)=\underset{y \in[0, f(x)]}{\operatorname{argmax}}\{u(f(x)-y)+\beta v(y)\} .
$$

It follows from Dechert and Nishimura (1983, Theorem 1) that the policy correspondence $K$ is strictly increasing in the following sense:

$$
\forall x \geq 0, \forall x^{\prime}>x, \forall y \in K(x), \forall y^{\prime} \in K\left(x^{\prime}\right), \quad y<y^{\prime}
$$

The following two lemmas are immediate consequences of (3.7).

Lemma 3.1. Any optimal path is strictly increasing, strictly decreasing, or stationary.

Lemma 3.2. Let $x_{0} \geq 0$ and $x_{0}^{\prime}>x_{0}$. Let $\left\{x_{t}\right\}$ and $\left\{x_{t}^{\prime}\right\}$ be optimal paths from $x_{0}$ and $x_{0}^{\prime}$, respectively. Then $x_{t}<x_{t}^{\prime}$ for all $t \in \mathbb{N}$.

Since $\lim _{c \downarrow 0} u^{\prime}(c)=\infty$, the nonnegativity constraints (2.3) are never binding as long as $x_{0}>0$. Thus

$$
\forall x>0, \forall y \in K(x), \quad 0<y<f(x)
$$

Furthermore, for any optimal path $\left\{x_{t}\right\}$ from $x_{0}>0$, the Euler equation holds for all $t \in \mathbb{Z}_{+}$:

$$
u^{\prime}\left(f\left(x_{t}\right)-x_{t+1}\right)=\beta u^{\prime}\left(f\left(x_{t+1}\right)-x_{t+2}\right) f^{\prime}\left(x_{t+1}\right) .
$$


This implies that any nonzero steady state $x>0$ satisfies $\beta f^{\prime}(x)=1$. To consider solutions to this equation, we define

$$
\beta_{I}=\frac{1}{f^{\prime}\left(x^{I}\right)}, \quad \beta_{0}=\frac{1}{\lim _{x \downarrow 0} f^{\prime}(x)} .
$$

We have $\beta_{I}<\beta_{0}$ since $f$ is strictly convex on $\left[0, x^{I}\right]$, but we do not know if $\beta_{0}$ or $\beta_{I}$ is less than one. The following result is an immediate consequence of (3.10) and Assumptions 2.3 and 2.4.

Lemma 3.3. (i) There exists a capital stock $x^{*} \geq x^{I}$ with $\beta f^{\prime}\left(x^{*}\right)=1$ if and only if $\beta \geq \beta_{I}$. If such $x^{*}$ exists, it is unique. (ii) There exists a capital stock $x_{*} \in\left(0, x^{I}\right]$ with $\beta f^{\prime}\left(x_{*}\right)=1$ if and only if $\beta \in\left[\beta_{I}, \beta_{0}\right)$. If such $x_{*}$ exists, it is unique.

For $x \geq 0$, define

$$
\gamma(x)=\beta f(x)-x .
$$

Kamihigashi and Roy (2007) call this the gain function. Provided that $x_{*}$ and $x^{*}$ exist and are distinct (i.e., $\beta_{I}<\beta<\beta_{0}$ ), we have

$$
\gamma^{\prime}(x) \begin{cases}<0 & \text { for } x \in\left(0, x_{*}\right) \\ =0 & \text { for } x=x_{*} \\ >0 & \text { for } x \in\left(x_{*}, x^{*}\right) \\ =0 & \text { for } x=x^{*} \\ <0 & \text { for } x>x^{*}\end{cases}
$$

If $x_{*}$ does not exist but $x^{*}$ does (i.e., $\beta_{0} \leq \beta<1$ ), then

$$
\gamma^{\prime}(x) \begin{cases}>0 & \text { for } x \in\left(0, x^{*}\right) \\ =0 & \text { for } x=x^{*} \\ <0 & \text { for } x>x^{*}\end{cases}
$$

We borrow the next four results from Kamihigashi and Roy (2007, Lemma 3.5, Propositions 3.2, 3.1, 4.2) and Dechert and Nishimura (1983, Theorem $2)$.

Lemma 3.4. Let $\left\{x_{t}\right\}$ be an optimal path that is not stationary. Then there exists $t \in \mathbb{N}$ such that $\gamma\left(x_{t}\right)>\gamma\left(x_{0}\right)$. 
Lemma 3.5. Let $y \geq 0$. If $y=\operatorname{argmax}_{x \geq 0} \gamma(x)$, then $y$ is a steady state.

Lemma 3.6. Any optimal path converges to a steady state.

Lemma 3.7. Suppose that $\lim _{x \downarrow 0} \gamma^{\prime}(x)<0$. Then there exists $y>0$ such that any optimal path from $x \in(0, y]$ converges to zero.

The next result is due to Dechert and Nishimura (1983, Lemma 3). We prove it here as a simple consequence of Lemma $3.4 .^{3}$

Lemma 3.8. Suppose that $x_{*}$ exists. Then there exists no optimal path from $x_{0} \in\left(0, x_{*}\right) \cup\left(x_{*}, \infty\right)$ that converges to $x_{*}$.

Proof. Suppose that there exists an optimal path $\left\{x_{t}\right\}$ from $x_{0} \in\left(0, x_{*}\right) \cup$ $\left(x_{*}, \infty\right)$ that converges to $x_{*}$. Since $x_{*}$ is a local minimizer of $\gamma$ by (3.12), it follows by Lemma 3.1 that $\left\{\gamma\left(x_{t}\right)\right\}_{t=i}^{\infty}$ is strictly decreasing for sufficiently large $i$. Since $\left\{x_{t}\right\}_{t=i}^{\infty}$ is optimal from $x_{i}$, this contradicts Lemma 3.4.

The next result combines the main results of Dechert and Nishimura (1983, Theorems 3, 4, 5) into a simple form. While Dechert and Nishimura (1983) consider three different cases based on the size of $\beta$, we consider only two cases based on whether or not $x^{*}$ is a steady state. Our result here allows for the possibility that $x^{c}=0$ or $x^{*}$. $^{4}$

Proposition 3.1. (i) Suppose that $x^{*}$ exists and is a steady state. Then there exists a critical capital stock $x^{c} \in\left[\underline{x}, x^{*}\right]$ such that any optimal path from $x \in\left(0, x^{c}\right)$ converges to zero and any optimal path from $x \in\left(x^{c}, \infty\right)$ converges to $x^{*}$. (ii) Suppose that $x^{*}$ does not exist or is not a steady state. Then any optimal path from $x>0$ converges to zero.

Proof. Let $\underline{y}=\sup \{x \geq 0$ : there exists an optimal path from $x$ that converges to zero $\}$. Note from (2.6) that

$$
\underline{x} \leq \underline{y}
$$

We claim that

if $\underline{y}>0$, any optimal path $\left\{x_{t}\right\}$ from $x \in(0, \underline{y})$ converges to zero.

\footnotetext{
${ }^{3}$ Since Dechert and Nishimura (1983, Lemma) only outline their proof, we prove Lemma 3.8 to confirm that it is valid under our assumptions.

${ }^{4}$ Proposition 4.1 identifies when such cases occur.
} 
To see this, note that there exists an optimal path $\left\{x_{t}^{\prime}\right\}$ from $x^{\prime} \in(x, y]$ that converges to zero. Thus by Lemma $3.2,\left\{x_{t}\right\}$ also converges to zero.

To prove part (i), let $\bar{y}=\inf \{x>0$ : there exists an optimal path from $x$ that converges to $\left.x^{*}\right\}$. We have $y \leq \bar{y} \leq x^{*}$ by Lemma 3.2. By the argument for (3.15), any optimal path from $x \in\left(\bar{y}, x^{*}\right)$ converges to $x^{*}$. Suppose that $\underline{y}<\bar{y}$. If $x_{*}$ exists, let $x \in(\underline{y}, \bar{y}) \backslash\left\{x_{*}\right\}$; if $x_{*}$ does not exist, let $x \in(\underline{y}, \bar{y})$. By definition of $y$ and $\bar{y}$ and Lemma 3.8, there is no optimal path from $x$ that converges to a steady state. Since this contradicts Lemma 3.6, we have $y=\bar{y}$. Letting $x^{c}=y\left(\geq \underline{x}\right.$ by (3.14)), we see that $x^{c}$ has the desired properties.

To prove part (ii), recall from Lemma 3.3 that if $x_{*}$ exists, then $x_{*} \leq x^{I}$. Thus any optimal path from $x>x^{I}$ converges to zero by Lemmas 3.6 and 3.8. This implies that $y=\infty$, and any optimal path from any $x>0$ converges to zero by (3.15).

For the rest of the paper, we let $x^{c}$ be the critical capital stock given by Proposition 3.1 provided that $x^{*}$ exists and is a steady state. We define

$$
\tilde{x}=\sup \{x>0: \forall y \in(0, x), \gamma(y)<0\} .
$$

If there is no $x>0$ such that $\gamma(y)<0$ for all $y \in(0, x)$, then we define $\tilde{x}=0$. We also define

$$
\begin{aligned}
& \hat{x}=\underset{x>0}{\operatorname{argmax}}\{f(x) / x\}, \\
& \hat{\beta}=\frac{1}{f(\hat{x}) / \hat{x}}=1 / f^{\prime}(\hat{x}) .
\end{aligned}
$$

Provided that $\tilde{x} \in(0, \infty)$ we have

$$
\underline{x}<\tilde{x} \leq \hat{x}<\bar{x}
$$

where $\underline{x}$ and $\bar{x}$ are defined in (2.5) and Assumption 2.4, respectively. The location of $x^{I}$ relative to $\underline{x}$ and $\tilde{x}$ is not clear, but since $f$ cannot be strictly convex on $[0, \hat{x}]$, we have

$$
x^{I}<\hat{x} .
$$

Recalling (3.10) we see that

$$
\beta_{I}<\hat{\beta}<\beta_{0}
$$

Lemma 3.9. (i) If $\hat{\beta} \geq 1$, then there exists no nonzero steady state. (ii) If $\hat{\beta}<1$ and $\beta \geq \hat{\beta}$, then $x^{*}$ exists and is a steady state. 
Proof. To see part (i), suppose that $\hat{\beta} \geq 1$. Let $x>0$. Then $f(x) / x \leq 1 / \hat{\beta} \leq$ 1, i.e., $f(x) \leq x$. Thus by (3.8) we have $y<f(x) \leq x$ for all $y \in K(x)$. Hence the conclusion follows. To see part (ii), note that existence of $x^{*}$ follows from Lemma 3.3(i) and (3.21). Since $1 / f^{\prime}\left(x^{*}\right)=\beta \geq \hat{\beta}=1 / f^{\prime}(\hat{x})$, we have $f^{\prime}\left(x^{*}\right) \leq f^{\prime}(\hat{x})$. Since $\hat{x}>x^{I}$ by $(3.20)$, it follows that $x^{*} \geq \hat{x}$ by concavity of $f$ on $\left[x^{I}, \infty\right)$. Thus by (3.12), (3.13), and (3.18), we have $\gamma\left(x^{*}\right) \geq \gamma(\hat{x}) \geq \hat{\beta} f(\hat{x})-\hat{x}=0$. Hence $x^{*}=\operatorname{argmax}_{x \geq 0} \gamma(x)$ by (3.12) and (3.13). This together with Lemma 3.5 shows that $x^{*}$ is a steady state.

Now we introduce two functions that play a key role in showing the continuity of the critical capital stock: for $x \geq 0$, define

$$
\begin{aligned}
v^{d}(x)=\max _{\left\{c_{t}, x_{t}\right\}_{t=0}^{\infty}} & \sum_{t=0}^{\infty} \beta^{t} u\left(c_{t}\right) \\
\text { s.t. } & c_{t}+x_{t+1}=f\left(x_{t}\right), \\
& c_{t}, x_{t+1} \geq 0 \\
& x_{t+1} \leq x_{t} \\
& x_{0}=x
\end{aligned}
$$

Note that $v^{d}(x)$ is the maximized value of the objective function under the additional constraint that feasible paths must be decreasing. ${ }^{5}$ We define $v^{i}(x)$ similarly by replacing (3.25) with

$$
x_{t+1} \geq \min \left\{x_{t}, f\left(x_{t}\right)\right\} .
$$

The right-hand side reflects the fact that it may not be feasible to choose $x_{t+1} \geq x_{t}$, since $x_{t}>f\left(x_{t}\right)$ if $x_{t}<\underline{x}$ or $x_{t}>\bar{x}$. However, since $\underline{x} \leq x^{c} \leq$ $x^{*}<\bar{x}$ by Proposition 3.1 and (3.8), we could restrict the domain of $v^{i}$ to $[\underline{x}, \bar{x}]$ without affecting the analysis of $x^{c}$. We do not do so here for notational convenience.

Lemma 3.10. (i) $v^{d}, v^{i}: \mathbb{R}_{+} \rightarrow \mathbb{R}_{+}$are continuous. (ii) Suppose that $x^{*}$ exists and is a steady state. Then

$$
\begin{aligned}
& \forall x \in\left(0, x^{c}\right) \cup\left(x^{*}, \infty\right), v^{d}(x)=v(x)>v^{i}(x), \\
& \forall x \in\left(x^{c}, x^{*}\right), \quad v^{d}(x)<v(x)=v^{i}(x) .
\end{aligned}
$$

\footnotetext{
${ }^{5}$ In this paper, "decreasing" means "nonincreasing," and "increasing" means "nondecreasing."
} 
Furthermore,

$$
v^{d}(x)=v^{i}(x)=v(x) \quad \Longleftrightarrow \quad x \in\left\{0, x^{c}, x^{*}\right\}
$$

Proof. Part (i) follows from a standard argument (e.g, Stokey and Lucas, 1989, Theorem 4.6). To see part (ii), note from Proposition 3.1, Lemma 3.1, and (3.8) that any optimal path from $x \in\left(0, x^{c}\right) \cup\left(x^{*}, \infty\right)$ is strictly decreasing with strictly positive consumption in each period. Thus $v^{d}(x)=$ $v(x)>v^{i}(x)$. Hence (3.28) follows. We obtain (3.29) similarly.

The $\Rightarrow$ part of (3.30) is immediate from (3.28) and (3.29). The $\Leftarrow$ part is also immediate if $x \in\left\{0, x^{*}\right\}$. Consider the case $x=x^{c}$. It follows from (3.28) and part (i) that $v^{d}(x)=v(x) \geq v^{i}(x)$. Likewise $v^{d}(x) \leq v(x)=v^{i}(x)$ by (3.29). Hence we obtain $v^{d}(x)=v(x)=v^{i}(x)$.

Lemma 3.11. Suppose that $x^{*}$ exists and is a steady state. (i) If $\tilde{x} \in(0, \infty)$, then $x^{c} \leq \tilde{x}$. (ii) If $\underline{x}>0$, then $\underline{x}<x^{c}$.

Proof. To see part (i), suppose that $\tilde{x} \in(0, \infty)$. Note from (3.12) that $x_{*}<\tilde{x}$ if $x_{*}$ exists. Since $\gamma^{\prime}(\tilde{x}) \geq 0$, we have $\tilde{x} \leq x^{*}$ by (3.12) and (3.13). If $\gamma\left(x^{*}\right)>0$, then by Lemmas 3.4, 3.6, and 3.8, any optimal path from $\tilde{x}$ converges to $x^{*}$. If $\gamma\left(x^{*}\right)=0$, then $\tilde{x}=x^{*}$. In either case, we have $x^{c} \leq \tilde{x}$.

To see part (ii), suppose that $\underline{x}>0$. Note from (3.8) that $y<f(\underline{x})=\underline{x}$ for any $y \in K(\underline{x})$. Thus by Lemmas 3.1, 3.6, and 3.8, any optimal path from $\underline{x}$ converges to zero, which implies that $v^{d}(\underline{x})>v^{i}(\underline{x})$. Since $v^{d}$ and $v^{i}$ are continuous, it follows that $\underline{x}<x^{c}$.

\subsection{Comparative Statics}

Since our main results are concerned with how the critical capital stock $x^{c}$ depends on $\beta$, in what follows we make explicit the dependence on $\beta$ of $v(x), v^{d}(x), x^{*}, x^{c}$, etc., by writing instead $v(x, \beta), v^{d}(x, \beta), x^{*}(\beta), x^{c}(\beta)$, etc. In addition, given $\beta \in(0,1)$, we call a capital path $\left\{x_{t}\right\}$ that is optimal for the maximization problem (2.1)-(2.4) $\beta$-optimal.

We start by observing (without proof) how $x^{*}(\beta), x_{*}(\beta)$, and $\tilde{x}(\beta)$ vary with $\beta$.

Lemma 3.12. (i) $x^{*}(\beta)$ is strictly increasing and continuous in $\beta \in\left[\beta_{I}, 1\right)$ with

$$
x^{*}\left(\beta_{I}\right)=x^{I}, \quad \lim _{\beta \uparrow 1} x^{*}(\beta)<\bar{x} .
$$


(ii) $x_{*}(\beta)$ is strictly decreasing and continuous in $\beta \in\left[\beta_{I}, \beta_{0}\right)$ with

$$
x_{*}\left(\beta_{I}\right)=x^{I}, \quad \lim _{\beta \uparrow \beta_{0}} x_{*}(\beta)=0 .
$$

(iii) If $\hat{\beta}<1$, then $\tilde{x}(\beta)$ is strictly decreasing and continuous in $\beta \in\left[\hat{\beta}, \beta_{m}\right)$ with

$$
\tilde{x}(\hat{\beta})=\hat{x}, \quad \lim _{\beta \uparrow \beta_{m}} \tilde{x}(\beta)=\underline{x},
$$

where

$$
\beta_{m}=\min \left\{\beta_{0}, 1\right\} .
$$

It follows from Amir et al. (1991, Theorem 5.5(d)) that the policy correspondence $K(x, \beta)$ is strictly increasing in $\beta$ for any $x>0$ :

$$
\forall \beta \in(0,1), \forall \beta^{\prime} \in(\beta, 1), \forall y \in K(x, \beta), \forall y^{\prime} \in K\left(x, \beta^{\prime}\right), \quad y<y^{\prime} .
$$

An immediate consequence of this is the following.

Lemma 3.13. Let $x>0$. Let $\beta \in(0,1)$ and $\beta^{\prime} \in(\beta, 1)$. Then for any $\beta$ optimal path $\left\{x_{t}\right\}$ from $x$ and $\beta^{\prime}$-optimal path $\left\{x_{t}^{\prime}\right\}$ from $x$, we have $x_{t}<x_{t}^{\prime}$ for all $t \in \mathbb{N}$.

The following result can be shown by a straightforward argument.

Lemma 3.14. For each $x>0, v(x, \beta), v^{d}(x, \beta)$, and $v^{i}(x, \beta)$ are strictly increasing and continuous in $\beta \in(0,1)$.

\section{Main Results}

In this section we state the main results of this paper, which are concerned with how the critical capital stock varies with $\beta$. Existence of a critical capital stock by definition assumes existence of a nonzero steady state. Therefore, to examine the properties of the critical capital stock, we need to assume that there exists a nonzero steady state at least for some $\beta \in(0,1)$. By Lemma 3.9 , a necessary and sufficient condition for this is the following.

Assumption 4.1. $\hat{\beta}<1$.

For the rest of this paper, we maintain Assumption 4.1 in addition to Assumptions 2.1-2.4. We are ready to state the first of our main results: 
Proposition 4.1. There exists $\underline{\beta} \in\left[\beta_{I}, \hat{\beta}\right]$ such that $x^{*}(\beta)$ exists and is a steady state if and only if $\beta \in[\bar{\beta}, 1)$. Furthermore, the critical capital stock $x^{c}(\beta)$ is decreasing and continuous in $\beta \in[\beta, 1)$.

Proof. See Section 5.

The monotonicity of $x^{*}(\cdot)$ in $\beta$ is a simple consequence of (3.35). The proof of the continuity of $x^{*}(\cdot)$ uses Lemmas 3.10 and 3.14 to derive a contradiction under the hypothesis that $x^{c}(\cdot)$ is discontinuous at some $\beta$.

The next result completes the picture by identifying the boundary values of $x^{c}(\cdot)$ :

Proposition 4.2. Let $\underline{\beta}$ and $\beta_{m}$ be given by Proposition 4.1 and (3.34), respectively. We have

$$
\forall \beta \in\left(\underline{\beta}, \beta_{m}\right), \quad \underline{x}<x^{c}(\beta)<x^{*}(\beta) .
$$

Furthermore,

$$
\begin{aligned}
x^{c}(\underline{\beta}) & =x^{*}(\underline{\beta}), \\
\lim _{\beta \uparrow \beta_{m}} x^{c}(\bar{\beta}) & =\underline{x} .
\end{aligned}
$$

If $\beta_{0}<1$, then

$$
\forall \beta \in\left[\beta_{0}, 1\right), \quad x^{c}(\beta)=0 .
$$

Proof. See Section 5.

Provided that $\beta_{0}<1$, Proposition 4.2 along with Propositions 4.1 and 3.1 shows that for all $\beta \in\left[\beta_{0}, 1\right)$, we have $x^{c}(\beta)=0$, and all optimal paths from $x>0$ converge to $x^{*}(\beta)$. For any $\beta \in\left(\beta, \beta_{0}\right)$, we have $x^{c}(\beta)>0$, and $x^{c}(\beta)$ increases to $x^{*}(\underline{\beta})$ as $\beta$ decreases to $\beta$. If $\beta$ decreases any further, both $x^{c}(\beta)$ and $x^{*}(\beta)$ disappear, and all optimal paths converge to zero.

\section{Proofs of Propositions 4.1 and 4.2}

We prove Propositions 4.1 and 4.2 after preparing several lemmas.

Lemma 5.1. Let $\beta, \beta^{\prime} \in\left[\beta_{I}, 1\right)$ be such that $\beta<\beta^{\prime}$ and both $x^{c}(\beta)$ and $x^{c}\left(\beta^{\prime}\right)$ exist. Then $x^{c}(\beta) \geq x^{c}\left(\beta^{\prime}\right)$. 
Proof. Suppose to the contrary that $x^{c}(\beta)<x^{c}\left(\beta^{\prime}\right)$. Fix $x \in\left(x^{c}(\beta), x^{c}\left(\beta^{\prime}\right)\right)$. Let $\left\{x_{t}\right\}$ be a $\beta$-optimal path from $x$, and $\left\{x_{t}^{\prime}\right\}$ be a $\beta^{\prime}$-optimal path from $x$. Since $x>x^{c}(\beta),\left\{x_{t}\right\}$ is strictly increasing. Since $x<x^{c}\left(\beta^{\prime}\right),\left\{x_{t}^{\prime}\right\}$ is strictly decreasing. But this contradicts Lemma 3.13.

Lemma 5.2. Let $\beta \in\left[\beta_{I}, 1\right)$ be such that $x^{*}(\beta)$ is a steady state. Then for any $\beta^{\prime} \in(\beta, 1), x^{*}\left(\beta^{\prime}\right)$ is a steady state.

Proof. Let $\beta^{\prime} \in(\beta, 1)$. Let $k: \mathbb{R}_{+} \rightarrow \mathbb{R}_{+}$be any selection from the policy correspondence $K\left(\cdot, \beta^{\prime}\right)$. Then $k\left(x^{*}(\beta)\right)>x^{*}(\beta)$ by $(3.35)$. Since $k(\cdot)$ is strictly increasing by (3.7), and since $k(\bar{x})<f(\bar{x})=\bar{x}$ by $(3.8), k(\cdot)$ has a fixed point in $\left[x^{*}(\beta), \bar{x}\right]$ by the Knaster-Tarski fixed point theorem (Aliprantis and Border, 1999, p. 15). If $x_{*}\left(\beta^{\prime}\right)$ exists, then $x_{*}\left(\beta^{\prime}\right)<x^{*}(\beta)$ by Lemma 3.12. Hence only $x^{*}\left(\beta^{\prime}\right)$ can be a fixed point of $k(\cdot)$ in $\left[x^{*}(\beta), \bar{x}\right]$. It follows that $x^{*}\left(\beta^{\prime}\right)$ is a fixed point of $k(\cdot)$, i.e., $x^{*}\left(\beta^{\prime}\right)$ is a steady state.

Lemma 5.3. There exists $\beta \in\left[\beta_{I}, \hat{\beta}\right]$ such that $x^{*}(\beta)$ is not a steady state for any $\beta \in\left[\beta_{I}, \underline{\beta}\right)$, and is a steady state for any $\beta \in[\underline{\beta}, 1)$.

Proof. Define $\beta=\inf \left\{\beta \in\left[\beta_{I}, \hat{\beta}\right]: x^{*}(\beta)\right.$ is a steady state $\}$. By (3.21) and Lemma 3.9(ii), $\beta$ exists. By definition of $\beta, x^{*}(\beta)$ is not a steady state for any $\beta \in\left[\beta_{I}, \beta\right)$. It follows from Lemma $5 . \overline{2}$ that $x^{*}(\beta)$ is a steady state for any $\beta \in(\beta, 1 \overline{)}$.

It remains to show that $x^{*}(\beta)$ is a steady state. To this end, suppose that $x^{*}(\beta)$ is not a steady state. Then by Proposition 3.1(ii), any optimal path from $x^{*}(\underline{\beta})$ converges to zero. Hence

$$
v^{d}\left(x^{*}(\underline{\beta}), \underline{\beta}\right)=v\left(x^{*}(\underline{\beta}), \underline{\beta}\right)>v^{i}\left(x^{*}(\underline{\beta}), \underline{\beta}\right) .
$$

Note from Proposition 3.1(i) that $x^{c}(\beta) \leq x^{*}(\beta)$ for all $\beta \in(\beta, 1)$. Since $x^{c}(\cdot)$ is decreasing by Lemma 5.1, and since $x^{*}(\cdot)$ is continuous and strictly increasing by Lemma 3.12(i), it follows that $x^{c}(\beta) \leq x^{*}(\underline{\beta})<x^{*}(\beta)$ for all $\beta \in(\underline{\beta}, 1)$. Thus by Lemma 3.10(ii),

$$
\forall \beta \in(\underline{\beta}, 1), \quad v^{d}\left(x^{*}(\underline{\beta}), \beta\right) \leq v\left(x^{*}(\underline{\beta}), \beta\right)=v^{i}\left(x^{*}(\underline{\beta}), \beta\right) .
$$

Letting $\beta \downarrow \underline{\beta}$ and recalling Lemma 3.14, we have $v^{d}\left(x^{*}(\underline{\beta}), \underline{\beta}\right) \leq v^{i}\left(x^{*}(\underline{\beta}), \underline{\beta}\right)$, which contradicts (5.1).

For the rest of this section we let $\underline{\beta} \in\left[\beta_{I}, \hat{\beta}\right]$ be given by Lemma 5.3. 
Lemma 5.4. $x^{c}(\beta)$ is continuous in $\beta \in[\underline{\beta}, 1)$.

Proof. Suppose that $x^{c}(\cdot)$ is discontinuous at some $\beta^{*} \in[\underline{\beta}, 1)$. This means that

$$
x^{c}\left(\beta^{*}\right)>\lim _{\beta \downarrow \beta^{*}} x^{c}(\beta)
$$

or that $\underline{\beta}<\beta^{*}$ and

$$
x^{c}\left(\beta^{*}\right)<\lim _{\beta \uparrow \beta^{*}} x^{c}(\beta) .
$$

Suppose that $\beta<\beta^{*}$ and (5.4) holds. Let $y=\lim _{\beta \uparrow \beta^{*}} x^{c}(\beta)$. Fix $x \in$ $\left(x^{c}\left(\beta^{*}\right), y\right)$. By Lemma 3.10 we have

$$
v^{d}\left(x, \beta^{*}\right)<v\left(x, \beta^{*}\right)=v^{i}\left(x, \beta^{*}\right) .
$$

Since $x<y \leq x^{c}(\beta)$ for all $\beta \in\left(\underline{\beta}, \beta^{*}\right)$, by Lemma 3.10 we have

$$
\forall \beta \in\left(\underline{\beta}, \beta^{*}\right), \quad v^{d}(x, \beta)=v(x, \beta)>v^{i}(x, \beta) .
$$

Letting $\beta \uparrow \beta^{*}$ and recalling Lemma 3.14, we obtain $v^{d}\left(x, \beta^{*}\right) \geq v^{i}\left(x, \beta^{*}\right)$, which contradicts (5.5). Hence (5.4) is ruled out. By a similar argument, (5.3) is also ruled out. It follows that $x^{c}(\cdot)$ is continuous.

Lemma 5.5. We have (4.1) in Proposition 4.2.

Proof. Note from Assumption 4.1 and (3.21) and (3.34) that $\hat{\beta}<\beta_{m}$, which implies $\beta<\beta_{m}$. Let $\beta^{\prime} \in\left(\beta, \beta_{m}\right)$ and $\beta \in\left(\beta, \beta^{\prime}\right)$. Then by Lemma 5.1, Proposition 3.1(i), and Lemma 3.12(i), we have

$$
x^{c}\left(\beta^{\prime}\right) \leq x^{c}(\beta) \leq x^{*}(\beta)<x^{*}\left(\beta^{\prime}\right) .
$$

If $\underline{x}>0$, then by Lemma 3.11(ii) we have

$$
\underline{x}<x^{c}\left(\beta^{\prime}\right) .
$$

Suppose that $\underline{x}=0$. Then $f^{\prime}(0) \equiv \lim _{x \downarrow 0} f^{\prime}(x) \geq 1$ by definition of $\underline{x}$. Thus $\beta_{0}=1 / f^{\prime}(0) \leq 1$, so that $\beta_{0}=\beta_{m}>\beta^{\prime}$. We have $\beta^{\prime} f^{\prime}(0)<\beta_{0} f^{\prime}(0)=1$. Hence by Lemma 3.7 we have $x^{c}\left(\beta^{\prime}\right)>0=\underline{x}$, i.e., (5.8) holds again.

It follows from (5.7) and (5.8) that $\underline{x}<x^{c}\left(\beta^{\prime}\right)<x^{*}\left(\beta^{\prime}\right)$. Since $\beta^{\prime} \in\left(\underline{\beta}, \beta_{m}\right)$ was arbitrary, we obtain (4.1).

Lemma 5.6. We have (4.2) and (4.3) in Proposition 4.2. 
Proof. To see (4.3), let $\beta \in\left(\hat{\beta}, \beta_{m}\right)$. Then $\underline{x}<\tilde{x}(\beta)<\hat{x}$ by Lemma 3.12(iii). From (4.1) and Lemma 3.11(i), we have $\underline{x}<x^{c}(\beta) \leq \tilde{x}(\beta)$. Thus (4.3) follows from Lemma 3.12(iii).

To see (4.2), let $y=x^{c}(\beta)$. Note from (4.1) that $y \leq x^{*}(\beta)$ since both $x^{c}(\cdot)$ and $x^{*}(\cdot)$ are continuous. Suppose that $y<x^{*}(\beta)$. Fix $\bar{x} \in\left(y, x^{*}(\beta)\right)$. For any $\beta \in(0, \beta)$, since $x^{*}(\beta)$ does not exist or is not a steady state by Lemmas 3.3(i) and 5.3, any $\beta$-optimal path from $x$ converges to zero by Proposition 3.1(ii). Thus

$$
\forall \beta \in(0, \underline{\beta}), \quad v^{d}(x, \beta)=v(x, \beta)>v^{i}(x, \beta) .
$$

Since $x>y \geq x^{c}(\beta)$ for all $\beta \in\left(\underline{\beta}, \beta_{m}\right)$ by Lemma 5.1 , we have

$$
\forall \beta \in\left(\underline{\beta}, \beta_{m}\right), \quad v^{d}(x, \beta)<v(x, \beta)=v^{i}(x, \beta) .
$$

Letting $\beta \rightarrow \beta$ in (5.9) and (5.10), it follows from Lemma 3.14 that $v^{d}(x, \beta)=$ $\left.v(x, \beta)=v^{i} \overline{(x}, \beta\right)$. Since this is true for any $x \in\left(y, x^{*}(\beta)\right)$, we have a contradiction to $(3.30)$.

\subsection{Proof of Proposition 4.1}

The first statement of the proposition follows from Lemmas 5.3 and 3.3. The second statement follows from Lemmas 5.1 and 5.4.

\subsection{Proof of Proposition 4.2}

We have (4.1)-(4.3) by Lemmas 5.5 and 5.6. Suppose that $\beta_{0}<1$. Then $\beta_{m}=\beta_{0}$ and $\underline{x}=0$. We obtain (4.4) from (4.3) since $x^{c}(\cdot)$ is decreasing and continuous by Proposition 4.1.

\section{References}

Aliprantis, C.D., Border, K.C., 1999, Infinite Dimensional Analysis: A Hitchhiker's Guide, Second Edition, Springer-Verlag, Berlin.

Amir, R., Mirman, L.J., Perkins, W.R., 1991, One-sector nonclassical optimal growth: optimality conditions and comparative dynamics, International Economic Review 32, 625-644. 
Caulkins, J.P., Feichtinger, G., Grass, D., Tragler, G., 2007, Bifurcating DNS thresholds in a model of organizational bridge building, Journal Optimization Theory and Applications 133, 19-35.

Caulkins, J.P., Hartl, R.F., Tragler, G., Feichtinger, G., 2001, Why politics makes strange bedfellows: dynamic model with DNS curves, Journal of Optimization Theory and Applications 111, 237-254.

Dechert, R., Nishimura, N., 1983, A complete characterization of optimal growth paths in an aggregated model with a non-concave production function, Journal of Economic Theory 31, 332-354.

Hartl, R.F., Kort, R.M., 2004, Optimal investments with convex-concave revenue: a focus-node distinction, Optimal Control Applications and Methods $25,147-164$.

Haunschmied, J.L., Feichtinger, G., Hartl, R.F., Kort, P.M., 2005, Keeping up with the technology pace: a DNS-curve and a limit cycle in a technology investment decision problem, Journal of Economic Behavior and Organization 57, 509-529.

Hung, N.M., Le Van, C., Michel, P., 2009, Non-convex aggregate technology and optimal economic growth, Economic Theory 40, 457-471.

Kamihigashi, T., Roy, S., 2007, A nonsmooth, nonconvex model of optimal growth, Journal of Economic Theory 132, 435-460.

Le Van, C., Morhaim, L., 2002, Optimal growth models with bounded or unbounded returns: a unifying approach, Journal of Economic Theory 105, 158-187.

Levy, A., Neri, F., Grass, D., 2006, Macroeconomic aspects of substance abuse: diffusion, productivity and optimal control, Macroeconomic Dynamics 10, 145-164.

Majumdar, M., Mitra, T., 1982, Intertemporal allocation with a non-convex technology: the aggregative framework, Journal of Economic Theory 27, 101-136.

Majumdar, M., Nermuth, M., 1982, Dynamic optimization in non-convex models with irreversible investment: monotonicity and turnpike results, Journal of Economics 42, 339-362.

Mitra, T., Ray, D., 1984, Dynamic optimization on a non-convex feasible set: some general results for non-smooth technologies, Journal of Economics 44, 151-174. 
Skiba, A.K., 1978, Optimal growth with a convex-concave production function, Econometrica 46, 527-539.

Stokey, N., Lucas, R.E., Jr., 1989, Recursive Methods in Economic Dynamics, Harvard University Press, Cambridge, MA.

Tragler, G., Caulkins, J.P., Feichtinger G., 2001, Optimal dynamic allocation of treatment enforcement in illicit drug control, Operations Research 49, $352-362$.

Wagener, F.O.O., 2005, Structural analysis of optimal investment for firms with non-concave revenue, Journal of Economic Behavior and Organization $57,474-489$.

Wirl, F., 2004, Thresholds in concave renewable resource models, Ecological Economics 48, 259-267. 